\title{
Influence of surface elasticity on the director profile of nontwisted nematic-liquid-crystal cells
}

\author{
S. Stallinga* \\ Institute for Theoretical Physics and Research Institute for Materials, University of Nijmegen Toernooiveld, \\ 6525 ED Nijmegen, The Netherlands
}

J.A.M.M. van Haaren and J.M.A. van den Eerenbeemd

Philips Research Laboratories, Prof. Holstlaan 4, 5656 AA Eindhoven, The Netherlands

(Received 22 March 1995)

\begin{abstract}
The Pergamenshchik theory [Phys. Rev. E 48, 1254 (1993)] and Faetti theory [Phys. Rev. E 49, $5332(1994) ; 49,4192(1994)]$ of surface elasticity in nematic liquid crystals are tested. Both theories give different predictions concerning the influence of the splay-bend surface elastic constant $K_{13}$ on the director profile of nontwisted liquid-crystal cells. The influence of $K_{13}$ on the director profile of nontwisted liquid-crystal cells is studied numerically within the framework of the Pergamenshchik theory. The capacitance and optical retardation of thin cells filled with the liquid crystal ZLI 4792 (E. Merck, Darmstadt, Germany) are measured as a function of the applied voltage. The surface tilt as a function of the applied voltage is calculated from these data and compared with numerically calculated curves. The model does not give a complete account of the observed optical behavior. Best agreement with the capacitive measurements is obtained with $K_{13}=0$. The effect of $K_{13}$ is comparable in magnitude with the effect of variations of the bulk liquid-crystal parameters within their experimental inaccuracy.
\end{abstract}

PACS number(s): 61.30.Eb, 62.20.Dc, 64.70.Md, 68.10.Cr

\section{INTRODUCTION}

Recent studies have improved our understanding of the interaction of nematic liquid crystals with the solid substrates that they are in contact with. In the past it was assumed that so-called strong anchoring applies to this interaction. This means that it was assumed that the orientation of the liquid-crystal molecules near the substrate is fixed along a certain preferential direction, irrespective of any external perturbations, such as magnetic or electric fields. Nowadays it is believed that so-called weak anchoring applies to the interaction of the liquid crystal with the solid substrate. It is assumed that this interaction still favors the alignment of the liquid-crystal molecules along the preferential direction, but that an external perturbation may cause the molecules to deviate from this preferred direction.

When the surface orientation of the liquid crystal is allowed to deviate from the preferential direction, the surface elasticity in the nematic liquid crystal must be taken into account as well. This subject has aroused considerable interest in recent years, mainly due to the fundamental difficulties caused by the introduction of the surface elastic constant $K_{13}[1]$. Currently there are two competing theories that offer a solution to this problem, the so-called Oldano-Barbero paradox. The first was put forward by Pergamenshchik [2], the second by Faetti [3]. The aim of this paper is to test both theories by studying the influence of surface elasticity on the director profile of nontwisted nematic-liquid-crystal cells. These cells are

${ }^{*}$ Present address: Philips Research Laboratories, Prof. Holstanlaan 4, 5656 AA, Eindhoven, The Netherlands. commonly referred to as ECB cells (ECB denotes electrically controlled birefringence) [4].

The dispute between Pergamenshchik and Faetti may be decided experimentally, as the predictions of both theories for ECB cells differ substantially. Faetti predicts that $K_{13}$ is of no importance for nontwisted cells, whereas Pergamenshchik predicts that $K_{13}$ is of substantial importance for nontwisted cells. An experimental determination of $K_{13}$ within the framework of the Pergamenshchik theory makes it possible to discriminate between the two theories: An experimental value $K_{13} \neq 0$ disagrees with the Faetti theory, whereas an experimental value $K_{13}=0$ disagrees with the Pergamenshchik theory, for it cannot be expected on microscopic grounds that $K_{13}$ is actually zero [5]. We developed a computer program based on [2] and compared its outcomes with experimental results.

It should be remarked that both theories of surface elasticity also give different predictions for nematic cells with a twisted director profile. However, we have two reasons for considering ECB-type cells. First, the absence of twist simplifies the problem mathematically, as the director tilt is the only variable that needs to be considered. The influence of surface elasticity on the calculated results will therefore be easier to identify. Secondly, the optical retardation of the passing light caused by the boundary layers must be added, instead of subtracted, as in nematic cells with a $90^{\circ}$ twist. This makes the ECB cell more sensitive to the optical detection of the nematic surface properties. Moreover, discrepancies between theoretical and experimental results concerning the electro-optical transmission of ECB cells have been reported [6]. In principle, the present analysis can be extended to twisted nematic structures, but the algebra required is considerably more complicated in that case. 


\section{THEORY}

The elastic part of the free energy density of a nematic liquid crystal is given by

$$
\begin{aligned}
f_{d}= & \frac{1}{2} K_{11}(\nabla \cdot n)^{2}+\frac{1}{2} K_{22}[n \cdot(\nabla \times n)]^{2} \\
& +\frac{1}{2} K_{33}[n \times(\nabla \times n)]^{2} \\
& +\frac{1}{2}\left(K_{22}+K_{24}\right) \nabla \cdot[(n \cdot \nabla) n-n(\nabla \cdot n)] \\
& +K_{13} \nabla \cdot[\boldsymbol{n}(\boldsymbol{\nabla} \cdot \boldsymbol{n})] .
\end{aligned}
$$

Here $n$ is the director and $K_{11}, K_{22}$, and $K_{33}$ are the bulk elastic constants for splay, twist, and bend, respectively [7], and $K_{24}$ and $K_{13}$ are the surface elastic constants for saddle splay and splay bend, respectively. Both surface contributions consist entirely of a divergence of a function of the director field and its derivatives and therefore only appear in the surface free energy density, as follows from Gauss's theorem. These surface terms have been introduced by Frank [8] and by Nehring and Saupe [9].

We will consider ECB cells with variations of the director field in the direction perpendicular to that of the cell substrates ( $z$ direction). For such an ECB cell, the director field is expressed as

$$
\boldsymbol{n}(z)=(\cos \theta(z), 0, \sin \theta(z)),
$$

where $\theta(z)$ is the tilt angle. This angle is the only variable for ECB cells. Then the total free energy (per unit of substrate area) is

$$
F=F_{s}+\int_{0}^{d} d z f
$$

where the free energy density is given by

$$
\begin{aligned}
f= & \frac{1}{2}\left(K_{11} \cos ^{2} \theta+K_{33} \sin ^{2} \theta\right) \theta^{\prime 2} \\
& +\frac{D^{2}}{2 \varepsilon_{0}\left(\varepsilon_{\perp} \cos ^{2} \theta+\varepsilon_{\|} \sin ^{2} \theta\right)}
\end{aligned}
$$

and the surface part of the free energy by

$$
\begin{aligned}
F_{s}= & \frac{1}{2} C_{\theta} \sin ^{2}\left[\theta(0)-\theta_{p}\right]+\frac{1}{2} C_{\theta} \sin ^{2}\left[\theta(d)-\theta_{p}\right] \\
& +\frac{1}{4} C_{2 \theta} \sin ^{4}\left[\theta(0)-\theta_{p}\right]+\frac{1}{4} C_{2 \theta} \sin ^{4}\left[\theta(d)-\theta_{p}\right] \\
& -\frac{1}{2} K_{13} \sin [2 \theta(0)] \theta^{\prime}(0)+\frac{1}{2} K_{13} \sin [2 \theta(d)] \theta^{\prime}(d) .
\end{aligned}
$$

Here $\theta^{\prime}$ is the spatial derivative of the tilt angle $\theta, D$ is the absolute value of the $z$ component of the displacement field, $\varepsilon_{\|}$and $\varepsilon_{\perp}$ are the dielectric constants of the nematic, $d$ is the thickness of the cell, $C_{\theta}$ is the RapiniPapoular anchoring constant [10], $C_{2 \theta}$ is the second-order anchoring constant, and $\theta_{p}$ is the pretilt angle. The elastic constants $K_{22}$ and $K_{24}$ do not appear, as the director field only depends on the tilt angle. The second-order anchoring constant $C_{2 \theta}$ is usually neglected, as it is only important when the deviation of the surface tilt angle from the pretilt angle is large, i.e., when the voltage is sufficiently above the Fréedericksz threshold.

The free energy including the $K_{13}$ term must be minimized with proper care. If the derivatives $\theta^{\prime}(0)$ and $\theta^{\prime}(d)$ are taken to be independent variables in the minimization procedure, an equilibrium director field with a discontinuity at the boundary is found. However, elasticity theory is based on the assumption that deformations are small. According to this assumption the higher-order elastic terms in the elastic free energy (all the terms with more than two spatial derivatives) may be neglected. The infinite deformations at the boundary are contradictory to the assumption of small deformations. This problem is called the Oldano-Barbero paradox [1].

In order to solve this paradox Pergamenshchik [2] assumed that the higher-order elastic terms stabilize the director field with respect to large deformations. According to Pergamenshchik the effect of higher-order terms can be taken into account by a constraint satisfied by the derivatives $\theta^{\prime}(0)$ and $\theta^{\prime}(d)$. The equilibrium director field is found by solving the usual Euler-Lagrange equations for fixed values of the surface tilt angles $\theta(0)$ and $\theta(d)$. With the aid of this solution $\theta^{\prime}(0)$ and $\theta^{\prime}(d)$ can be expressed in terms of $\theta(0)$ and $\theta(d)$. These expressions are then substituted in the surface free energy [Eq. (5)]. The resulting total free energy must be minimized with respect to $\theta(0)$ and $\theta(d)$ in order to obtain the equilibrium values of the surface tilt angles. These equilibrium values then give the equilibrium director field. It was shown by Faetti that the Pergamenshchik solution to the OldanoBarbero paradox causes a problem in the balancing of the various torques acting on the nematic $[3,11]$.

According to Faetti [3] the part of the splay-bend surface term that depends on the normal derivatives of the surface tilt angles $\theta^{\prime}(0)$ and $\theta^{\prime}(d)$ and the stabilizing higher-order elastic terms induce a strong subsurface deformation, i.e., a deformation of the director field in a microscopically thin region near the boundary. As far as the macroscopic variation of the director field is concerned, the part of the splay-bend surface term that depends on the normal derivatives of the surface tilt angles and the higher-order terms can be regarded as a contribution to the anchoring energy. In the case of the presently considered ECB cells the total splay-bend term depends on $\theta^{\prime}(0)$ and $\theta^{\prime}(d)$. Therefore, according to Faetti the conventional theory without the $K_{13}$ term is correct for ECB cells. Consequently, the Faetti theory for the ECB cell is equivalent to the Pergamenshchik theory in the special case of $K_{13}=0$. As opposed to the Pergamenshchik theory, the Faetti theory does not violate the balance of torques.

Neither approach is entirely satisfying. The theory of Pergamenshchik disagrees with the requirement for mechanical equilibrium, as a net torque acting on the liquidcrystal cell is predicted. The theory of Faetti predicts a microscopic phenomenon such as the strong subsurface deformation, which cannot be described by continuum theory.

The relevant equations for the calculation of the director field within the framework of the Pergamenshchik theory are given below. The anchoring is assumed to be symmetric under a reflection in the plane $z=d / 2$. Consequently, the director profile is assumed to have this symmetry as well, i.e., 


$$
\theta(z)=\theta(d-z)
$$

The Euler-Lagrange equation can be integrated twice with the aid of this symmetry relation. The following equation is obtained:

$$
\frac{d}{2}=\int_{\theta_{s}}^{\theta_{m}} d \theta\left[\frac{1-\gamma \cos ^{2} \theta}{\left(\cos ^{2} \theta-\cos ^{2} \theta_{m}\right) g\left(\theta, \theta_{m}\right)}\right]^{1 / 2},
$$

where $\theta_{s}=\theta(0)=\theta(d)$ is the surface tilt angle, $\theta_{m}=$ $\theta(d / 2)$ is the tilt angle in the middle of the cell, which is also the maximum tilt angle, and $\gamma=\left(K_{33}-K_{11}\right) / K_{33}$. The function $g\left(\theta, \theta_{m}\right)$ is defined by

$$
g\left(\theta, \theta_{m}\right)=\frac{D^{2} \alpha}{\varepsilon_{0} \varepsilon_{\|} K_{33}\left(1-\alpha \cos ^{2} \theta\right)\left(1-\alpha \cos ^{2} \theta_{m}\right)},
$$

where $\alpha=\left(\varepsilon_{\|}-\varepsilon_{\perp}\right) / \varepsilon_{\|}$. The applied voltage can be expressed as

$$
\begin{aligned}
\frac{V}{2}= & \frac{D}{\varepsilon_{0} \varepsilon_{\|}} \int_{\theta_{s}}^{\theta_{m}} d \theta\left[\frac{1-\gamma \cos ^{2} \theta}{\left(\cos ^{2} \theta-\cos ^{2} \theta_{m}\right) g\left(\theta, \theta_{m}\right)}\right]^{1 / 2} \\
& \times \frac{1}{1-\alpha \cos ^{2} \theta} .
\end{aligned}
$$

Equations (7) and (9) define $\theta_{m}$ and $D$ as functions of $\theta_{s}$ and the applied voltage $V$. The surface tilt $\theta_{s}$ can be solved as a function of $V$ from the condition that the total free energy is minimal:

$$
\frac{d F}{d \theta_{s}}=0
$$

Using the Euler-Lagrange equation this condition can be expressed as the Pergamenshchik boundary condition

$\begin{aligned} C_{\theta} \sin \left(\theta_{s}-\theta_{p}\right) \cos \left(\theta_{s}-\theta_{p}\right)+C_{2 \theta} \sin ^{3}\left(\theta_{s}-\theta_{p}\right) \cos \left(\theta_{s}-\theta_{p}\right) & \\ = & {\left[\left(K_{33}-K_{13}\right) \sin ^{2} \theta_{s}+\left(K_{11}+K_{13}\right) \cos ^{2} \theta_{s}\right] \theta^{\prime}(0)+\frac{1}{2} K_{13} \sin [2 \theta(0)] \frac{d \theta^{\prime}(0)}{d \theta_{s}} . }\end{aligned}$

The Faetti boundary condition is obtained by setting $K_{13}=0$. Taking $K_{13}$ into account requires the calculation of the derivative of $\theta^{\prime}(0)$ with respect to $\theta_{s}$. An analytical expression for this derivative is derived in Appendix A. This derivative can also be calculated numerically, but the analytical treatment proved to give better results.

The surface tilt $\theta_{s}$ is solved iteratively from Eq. (11). Combining Eqs. (8) and (9), an equation is obtained without the dielectric displacement $D$. In each iterative step the maximum tilt $\theta_{m}$ is solved from this equation. The variable $D$ can then be obtained from Eq. (7) by numerical integration.

The relevant electro-optical properties, i.e., the capacitance and the optical retardation, can now be calculated [6]. The capacitance per unit of substrate area is $D / V$ and the optical retardation is given by

$$
\begin{aligned}
\delta= & \frac{4 \pi}{\lambda} \int_{\theta_{s}}^{\theta_{m}} d \theta\left[\frac{1-\gamma \cos ^{2} \theta}{\left(\cos ^{2} \theta-\cos ^{2} \theta_{m}\right) g\left(\theta, \theta_{m}\right)}\right]^{1 / 2} \\
& \times\left(\frac{n_{\mathrm{o}} n_{\mathrm{e}}}{\sqrt{n_{\mathrm{o}}^{2} \cos ^{2} \theta+n_{\mathrm{e}}^{2} \sin ^{2} \theta}}-n_{o}\right)
\end{aligned}
$$

where $\lambda$ is the wavelength of the light and $n_{\mathrm{o}}$ and $n_{\mathrm{e}}$ are the ordinary and extraordinary indices of refraction, respectively.

\section{NUMERICAL RESULTS}

A computer program was developed that calculates the director profile as a function of the applied voltage. To this aim, Eqs. (7), (9), and (11) were solved with the aid of routines from the NAG mathematical library. A typical calculation took about ten seconds CPU time on a HP9000/735 with a HP-UX 9.01 operating system. Unless stated otherwise, the parameters in the calculations were $d=3.0 \mu \mathrm{m}, \theta_{p}=2.0^{\circ}, C_{\theta}=100 \mu \mathrm{J} / \mathrm{m}^{2}, C_{2 \theta}=0 \mu \mathrm{J} / \mathrm{m}^{2}$, $\varepsilon_{\|}=7.9, \varepsilon_{\perp}=3.1, \lambda=632.8 \mathrm{~nm}, n_{\mathrm{e}}=1.573, n_{\mathrm{o}}=$ $1.477, K_{11}=10 \mathrm{pN}, K_{33}=15 \mathrm{pN}, K_{13}=2 \mathrm{pN}$.

The influence of variations in $K_{13}$ on the tilt angles can be formulated as follows. At low voltages the surface tilt increases if $K_{13}$ is increased, whereas at high voltages the surface tilt decreases if $K_{13}$ is increased. A qualitative explanation of this behavior is given in Appendix B. The surface tilt is a concave function of the applied voltage in the region just above the threshold voltage for $K_{13}>0$ and a convex function of the applied voltage in the region just above the threshold for $K_{13}<0$. The tilt in the middle of the cell shows little sensitivity to variations in $K_{13}$. This is illustrated in Fig. 1 , where $\theta_{s}$ and $\theta_{m}$ are plotted for various values of $K_{13}$. The consequences for the capacitance relative to its saturation value and the optical retardation relative to its value at zero voltage are shown in Fig. 2. The influence of variations in $K_{13}$ is of the same order of magnitude as the influence of variations in the bulk elastic constants $K_{11}$ and $K_{33}$.

The surface elastic constant $K_{13}$ is only relevant in 


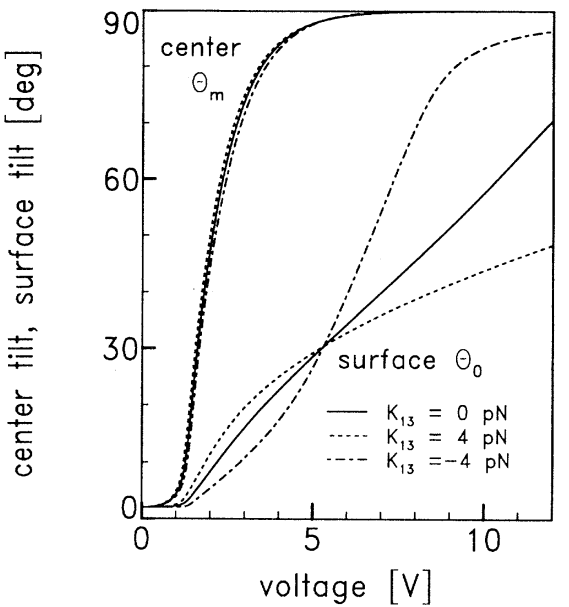

FIG. 1. Calculated results for the tilt in the center of the cell $\theta_{m}$ and at the surface $\theta_{s}$ for various values of $K_{13}$.

the regime of weak anchoring, i.e., in the regime where $C_{\theta} d / K=O(1)$ ( $K$ is a typical elastic constant). In the limit $C_{\theta} \rightarrow \infty$ the influence of $K_{13}$ is negligible. For cells with the standard setting of the parameters a value of $C_{\theta}=O\left(10^{5} \mu \mathrm{J} / \mathrm{m}^{2}\right)$ is sufficient for all weak anchoring effects to become negligible. The approach of the strong anchoring limit can also be studied by varying $d$ instead of $C_{\theta}$. However, in that case the optical retardation is not a convenient measure, since it contains a factor $d / \lambda$.

The influence of $K_{13}$ can be compared with the influence of the $C_{2 \theta}$ term in the anchoring energy. In Fig. 3 the surface tilt angle $\theta_{s}$ and the maximum tilt angle $\theta_{m}$ are plotted for various values of $K_{13}$ and $C_{\theta}$ so that the same Fréedericksz threshold applies to the different curves. In all cases $C_{2 \theta}=0$ was taken. In Fig. 4

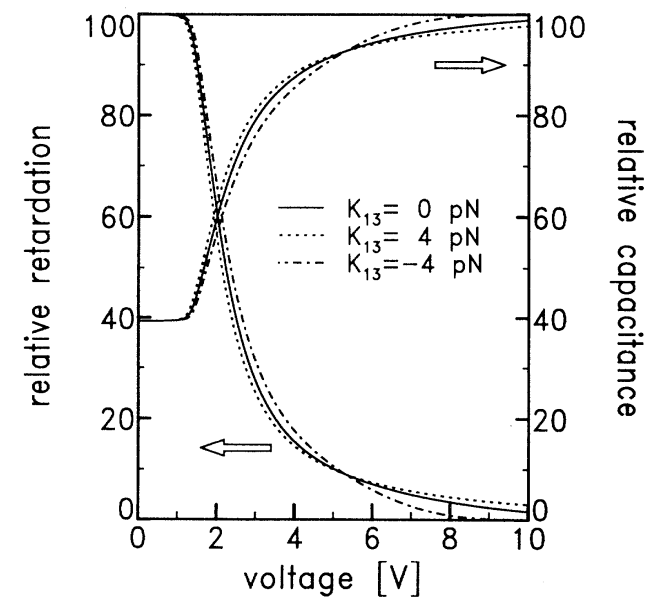

FIG. 2. Calculated results for the capacitance relative to its saturation value and the optical retardation relative to its value at zero voltage for $C_{\theta}=100 \mu \mathrm{J} / \mathrm{m}^{2}$ and various values of $K_{13}$. The arrows connect the lines with the relevant axes.
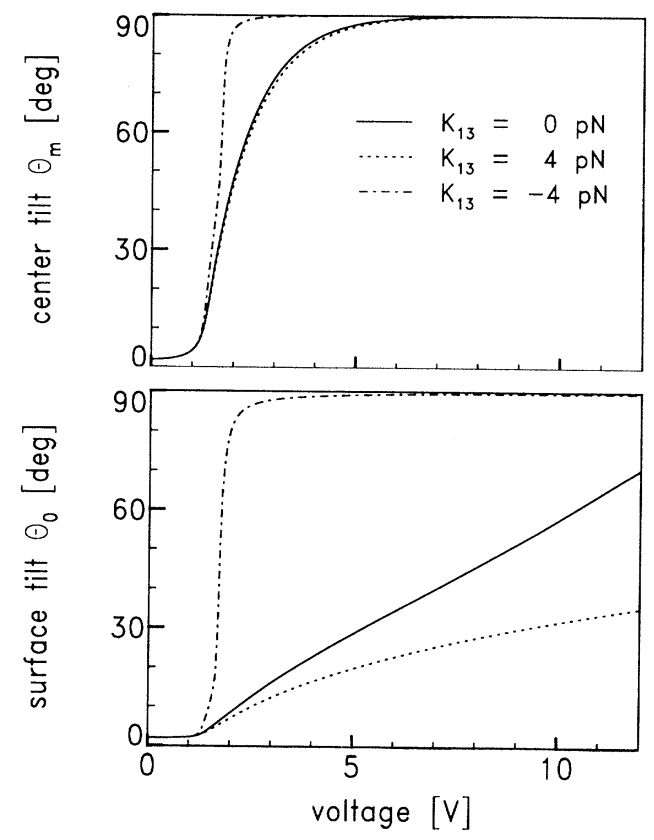

FIG. 3. Calculated results for the tilt at the center of the cell $\theta_{m}$ and at the surface $\theta_{s}$ for various values of $K_{13}$ and $C_{\theta}$. These parameters were varied such that the same Fréedericksz threshold held for the various curves. The lines pertain to $K_{13}=-4 \mathrm{pN}$ and $C_{\theta}=20 \mu \mathrm{J} / \mathrm{m}^{2}$, to $K_{13}=0 \mathrm{pN}$ and $C_{\theta}=100 \mu \mathrm{J} / \mathrm{m}^{2}$ and to $K_{13}=4 \mathrm{pN}$ and $C_{\theta}=180 \mu \mathrm{J} / \mathrm{m}^{2}$.

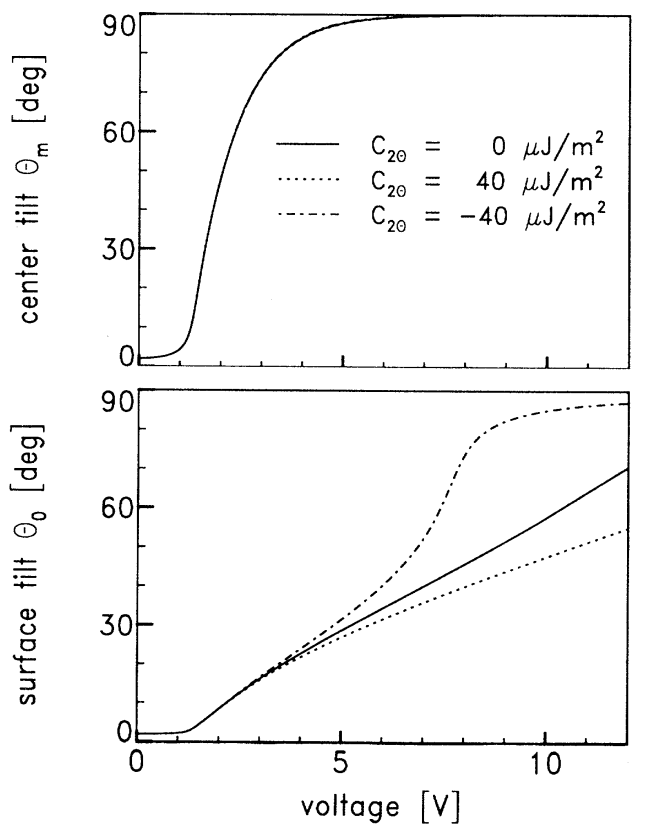

FIG. 4. Calculated results for the tilt at the center of the cell $\theta_{m}$ and at the surface $\theta_{s}$ for various values of $C_{2 \theta}$. For all curves $K_{13}=0$ and $C_{\theta}=100 \mu \mathrm{J} / \mathrm{m}^{2}$ were taken. 
the surface and maximum tilt angles $\theta_{s}$ and $\theta_{m}$ are plotted for various values of $C_{2 \theta}$. In all cases $K_{13}=0$ and $C_{\theta}=100 \mu \mathrm{J} / \mathrm{m}^{2}$ were taken. Note that for a given value of the Fréedericksz threshold the slope of the $\theta_{s}$ curve just above threshold is substantially altered by a variation in $K_{13}$, but not by a variation in $C_{2 \theta}$. To be able to use this fact for an experimental determination of $K_{13}$, the difference between the strong anchoring threshold and the weak anchoring threshold (see Appendix B) must be considerable, i.e., the anchoring constant $C_{\theta}$ must not be too large.

When $K_{13}<-K_{11} / 2$ or $K_{13}>K_{33} / 2$ the numerical results show unusual behavior, e.g., a surface tilt smaller than the pretilt at low voltages when $K_{13}<-K_{11} / 2$. This behavior is related to the predictions of Pergamenshchik, Teixeira, and Sluckin [12]. It probably implies that an antisymmetric director profile has lower free energy than the symmetric one. This means that our analysis is not applicable to cases with these values of $K_{13}$, as the director profile is assumed to be symmetric.

The influence of the pretilt angle on the Fréedericksz transition and the saturation transition $[13,14]$ is shown in Figs. 5 and 6, where $\theta_{s}$ and $\theta_{m}$, respectively, are plotted for various values of $\theta_{p}$. Within the Rapini-Papoular approximation of the anchoring energy these transitions can only be sharply defined when $\theta_{p}=0[15,16]$. This is due to the fact that the anchoring energy is minimal for a surface tilt equal to 0 and maximal for a surface tilt equal to $\pi / 2$ if and only if $\theta_{p}=0$. A quantitative argument is given in Appendix B. The Rapini-Papoular approximation is not correct close to saturation. Our results for the saturation regime can therefore only be in qualitative agreement with the experimental results.

\section{EXPERIMENT}

Optical and dielectric measurements were carried out on liquid-crystal test cells in order to compare the cal-

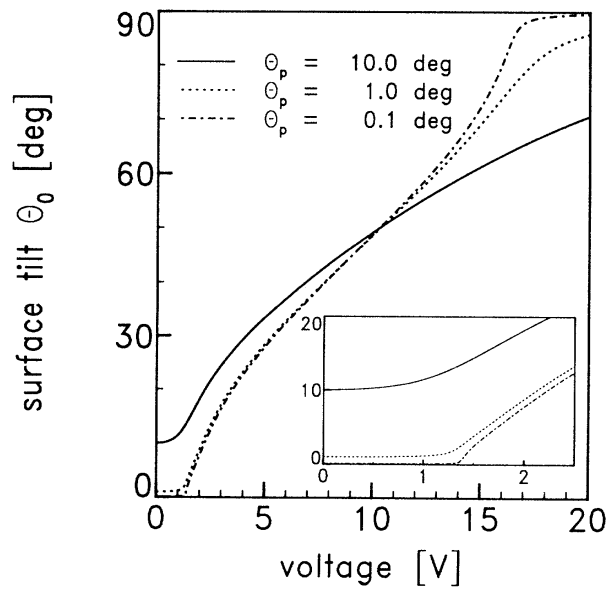

FIG. 5. Calculated results for the tilt at the surface $\theta_{s}$ for various values of the pretilt $\theta_{p}: 10.0^{\circ}, 1.0^{\circ}$, and $0.1^{\circ}$.

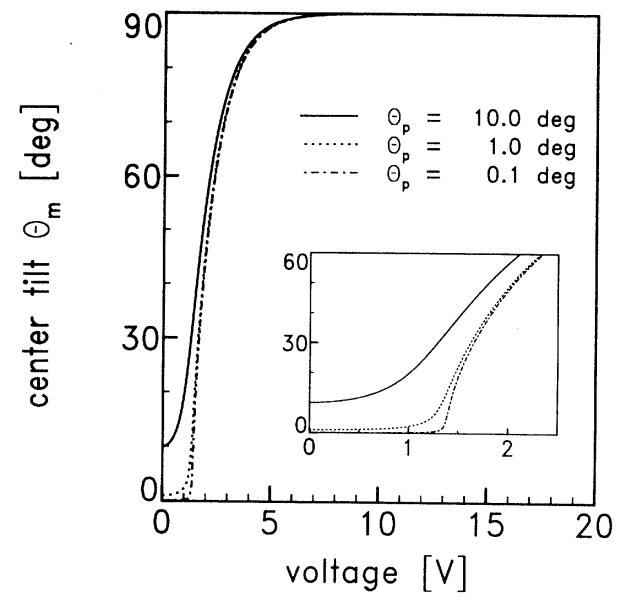

FIG. 6. Calculated results for the tilt at the center of the cell $\theta_{m}$ for pretilt values equal to $10.0^{\circ}, 1.0^{\circ}$, and $0.1^{\circ}$.

culated voltage dependence of the surface tilt angle $\theta_{s}$ with the response of real liquid-crystal cells. The measurements were done on nontwisted liquid-crystal cells filled with the superfluorinated liquid-crystal material ZLI 4792 (supplied by E. Merck, Darmstadt, Germany). This material melts below $-40^{\circ} \mathrm{C}$ and has its clearing point at $92^{\circ} \mathrm{C}$. Our measurements were performed at $20^{\circ} \mathrm{C}$. The ZLI 4792 material was selected because of its low ionic impurity content, which guarantees a welldefined transfer of the applied voltage to the liquidcrystal layer. Cells with thicknesses of $2.82 \mu \mathrm{m}, 3.76$ $\mu \mathrm{m}, 5.88 \mu \mathrm{m}$, and $14.74 \mu \mathrm{m}$ were examined. The liquidcrystal cells contained transparent conductive electrodes and thin $(110 \mathrm{~nm})$ buffed polyimide alignment layers (AL 1051, supplied by JSR, Kawasaki, Japan). A polyimide was chosen for our experiment, because these alignment materials are well suited for electro-optical experiments on liquid-crystal cells. The reason for this is that they combine good alignment properties with a low ionic contamination of the liquid-crystal material. The AL 1051 polyimide was selected, because it is a commercially available material with physical and chemical properties that have been published $[17,18]$.

The experiments consisted of an accurate measurement of the voltage dependence of the optical retardation for $632.8 \mathrm{~nm}$ wavelength laser light and of the capacitance for an electrode area of $0.50 \mathrm{~cm}^{2}$, driven by a $1 \mathrm{kHz}$ sine wave voltage. Typical results for a cell with $2.82 \mu \mathrm{m}$ thickness are shown in Fig. 7.

The optical retardation was measured in a setup as described by van Sprang [19]. In this setup linearly polarized light is incident on a liquid-crystal cell with its extraordinary axis under a $45^{\circ}$ angle with respect to the polarization direction of the light. After passing the liquidcrystal layer the light is elliptically polarized. A quarterwave retarder is used to make the light linearly polarized again, but now the polarization direction is rotated with respect to the incident light. The light passes a rotating analyzer, after which the intensity is measured. The same 


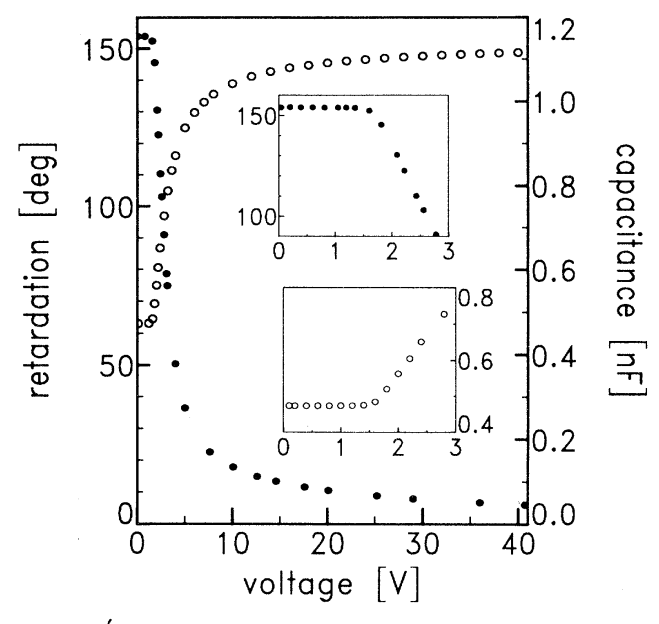

FIG. 7. Typical measurement results for the cell with a $2.82 \mu \mathrm{m}$ cell gap. Open symbols correspond to the measured capacitance, filled symbols to the measured retardation.

is done for a reference beam that does not pass the liquidcrystal layer and the quarter-wave retarder. The phase difference between the temporally varying intensities of these two beams is measured accurately. The polarization direction of the reference beam that is incident on the rotating analyzer is adjusted in such a way that the phase difference between the two beams is zero for a setup without a liquid-crystal cell in the primary beam. The phase difference measured in this way is equal to the optical retardation $\delta$ that was introduced in Eq. (12). This phase difference was measured as a function of the rms value of a $1 \mathrm{kHz}$ sine wave voltage. The typical accuracy of these measurements is within $0.1^{\circ}$.

The capacitance was measured using a General Radio 1621 Capacitance-Measurement System (accuracy $0.05 \%$ ) which consists of a precision capacitor bridge, an oscillator, and a detector. The measurements were done with sine wave voltages of $1 \mathrm{kHz}$ frequency. It was checked that the effect of an increase in the measuring frequency to $10 \mathrm{kHz}$ was small.

The surface tilt angle $\theta_{s}$ and the tilt angle at the middle of the cell $\theta_{m}$ at each value of the applied voltage were calculated from the measured data as follows [20]. The $z$ component of the dielectric displacement $D$ directly follows from the measured capacitance. Then the remaining unknowns, the surface tilt angle and the tilt angle at the middle of the cell, can be solved with the aid of Eqs. (7) and (9). The other parameters that serve as input for the numerical procedure are the bulk liquidcrystal material properties - elastic constants, dielectric constants and refractive indices - the pretilt angle and the cell gap. These parameters were measured separately, and are therefore not adjustable in the fitting procedure. The surface tilt can be determined with an accuracy of $4 \%$, following this procedure. Sources of inaccuracy are the errors in the capacitance measurement, in the determination of the Fréedericksz threshold from which the elastic constants are derived, and in the determination of the ratio of the splay and bend elastic constants. When the optical retardation is measured, the tilt angles can be solved with the aid of Eqs. (9) and (12).

The liquid-crystal material constants and the cell parameters were determined as follows. The thickness and the pretilt angle were measured with a setup and a method as described in [19]. The dielectric constant of the polyimide was $\varepsilon_{\text {pol }}=2.6$ [17]. The dielectric constant $\varepsilon_{\perp}$ of the liquid crystal was calculated from the capacitance at zero voltage $C_{0}$ using

$$
\varepsilon_{\perp}=d\left(\frac{\varepsilon_{0} A}{C_{0}}-\frac{2 d_{\mathrm{pol}}}{\varepsilon_{\mathrm{pol}}}\right)^{-1}
$$

where $A$ is the electrode area and $d_{\text {pol }}$ the thickness of the polyimide alignment layers. The pretilt angle is neglected in this formula. The dielectric constant $\varepsilon_{\|}$was derived from the linear extrapolation of the measured capacitance to infinite voltage $C_{\infty}$. This value was derived from a plot of the measured capacitance as a function of $1 / V$, where $V$ is the applied voltage. However, this plot turns out to be curved instead of linear at high voltages. This was also observed in the numerical results obtained with the computer program described in Sec. II. As a consequence, $\varepsilon_{\|}^{\infty}$, the value for $\varepsilon_{\|}$calculated from $C_{\infty}$, tends to be an overestimate of the true $\varepsilon_{\|}$. This was also concluded in [21]. The surface tilt appeared to be a decreasing function of the applied voltage at high voltages when $\varepsilon_{\|}$was taken to be equal to $\varepsilon_{\|}^{\infty}$. For the test cell with a $14.74 \mu \mathrm{m}$ cell gap, negative surface tilt values occurred in this range of voltages. Various values of $\varepsilon_{\|}$ between $0.5 \%$ and $2.5 \%$ below $\varepsilon_{\|}^{\infty}$ were used to calculate the surface tilt as a function of the applied voltage. The lower bound corresponds to the measured capacitance at the highest voltage, which was approximately $2.5 \%$ lower than $C_{\infty}$. The upper bound corresponds to the highest value of $\varepsilon_{\|}$for which the surface tilt is an increasing function of the applied voltage.

The values of the indices of refraction (at $632.8 \mathrm{~nm}$ wavelength) $n_{o}=1.477$ and $n_{e}=1.573$ were provided by the manufacturer of the liquid-crystal material [22]. The refractive index $n_{e}$ was also fitted to the retardation at zero voltage,

$$
\delta_{0}=\frac{2 \pi d n_{o}}{\lambda}\left(\frac{n_{e}}{\sqrt{n_{o}^{2} \cos ^{2} \theta_{p}+n_{e}^{2} \sin ^{2} \theta_{p}}}-1\right) .
$$

The result was in agreement with the data provided.

The splay elastic constant $K_{11}$ was calculated from the Fréedericksz threshold $V_{\mathrm{Fr}}=(1.58 \pm 0.02) \mathrm{V}$ using

$$
K_{11}=\varepsilon_{0}\left(\varepsilon_{\|}-\varepsilon_{\perp}\right)\left(\frac{V_{\mathrm{Fr}}}{\pi}\right)^{2} .
$$

The difference between the threshold voltage for weak anchoring and the threshold voltage for strong anchoring was usually smaller than $0.01 \mathrm{~V}$, as the anchoring constant appeared to be quite high. Hence this difference is neglected in the determination of the splay elastic con- 
stant $K_{11}$. The bend elastic constant $K_{33}$ follows from $K_{11}$ and the ratio $K_{11} / K_{33}=0.72 \pm 0.01$. This ratio was obtained from the values $K_{11}=13.2 \mathrm{pN}$ and $K_{33}=18.3$ $\mathrm{pN}$ at $20^{\circ} \mathrm{C}$ as provided by the liquid-crystal material manufacturer [22].

\section{RESULTS AND DISCUSSION}

In this section, the experimentally determined voltage dependence of the surface tilt is compared with the theoretical results obtained with the computer program described in Sec. II. The surface tilt is chosen for this comparison, as the numerical results in Sec. III indicate that the surface tilt is quite sensitive to variations in $K_{13}$ and thus the most suitable variable to be used for the comparison with theory. Figure 8 shows the results for the cell with the $2.82 \mu \mathrm{m}$ cell gap and $\varepsilon_{\|}=9.103$, which is $2 \%$ below $\varepsilon_{\|}^{\infty}=9.289$. The data points were calculated from the measured capacitances. The inaccuracy in the data is $4 \%$, which is roughly the size of the circles. The theoretical curves were calculated for the same values for the cell gap, dielectric constants, and bulk elastic constants as those used in the evaluation of the data points. The surface tilt angle below the Fréedericksz threshold agrees with the independently measured pretilt angle $\theta_{p}=1.07 \pm 0.10^{\circ}$. The different curves were calculated with different values for the splay-bend elastic constant $K_{13}$, namely, $0 \mathrm{pN},-4 \mathrm{pN}$, and $4 \mathrm{pN}$. The anchoring constant $C_{\theta}$ was adjusted for each value of $K_{13}$ to give an optimum agreement with the data points $\theta_{s}$ below $20^{\circ}$, as for these surface tilt values the Rapini-Papoular approximation is expected to be valid.

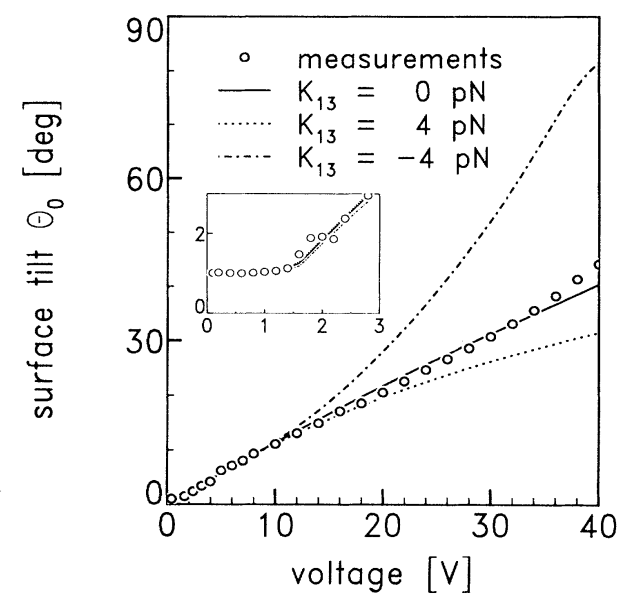

FIG. 8. Results for the tilt at the surface $\theta_{s}$ as calculated from the measured capacitance data for the cell with a 2.82 $\mu \mathrm{m}$ cell gap. The other parameters were $K_{11}=13.0 \mathrm{pN}$, $K_{33}=18.0 \mathrm{pN}, \varepsilon_{\perp}=3.309, \varepsilon_{\|}=9.103$. The curves give the results for the surface tilt as a function of the applied voltage for different values of $K_{13}$ and $C_{\theta}$, namely, for $K_{13}=4 \mathrm{pN}$ and $C_{\theta}=770 \mu \mathrm{J} / \mathrm{m}^{2}$, for $K_{13}=0 \mathrm{pN}$ and $C_{\theta}=600 \mu \mathrm{J} / \mathrm{m}^{2}$, and for $K_{13}=-4 \mathrm{pN}$ and $C_{\theta}=440 \mu \mathrm{J} / \mathrm{m}^{2}$.
The effect of a variation in $\varepsilon_{\|}$within the experimental uncertainty of this parameter, as discussed in Sec. IV, is shown in Fig. 9. This figure depicts the voltagedependent surface tilt of the $2.82 \mu \mathrm{m}$ thick cell, as calculated from the measured data for four different values of $\varepsilon_{\|}$: the upper and lower bounds for $\varepsilon_{\|}$, which are $0.5 \%$ and $2.5 \%$ below $\varepsilon_{\|}^{\infty}$, and two intermediate values $1.0 \%$ and $2.0 \%$ below $\varepsilon_{\|}^{\infty}$. The surface tilt as a function of the applied voltage depends strongly on the value chosen for $\varepsilon_{\|}$, despite the fact that the relative difference between the upper and lower bounds for $\varepsilon_{\|}$is only $2 \%$.

Such a variation in $\varepsilon_{\|}$also has a substantial effect on the theoretical curves. It is important to note that the theoretical curves must be calculated with the same value for $\varepsilon_{\|}$-and the remaining material parameters - as were used for the evaluation of the surface tilt from the measured capacitance data. Figure 10 shows the data points and numerical fits for the cell with a thickness $2.82 \mu \mathrm{m}$ for $\varepsilon_{\|}=9.196$, which is $1 \%$ below $\varepsilon_{\|}^{\infty}$. This value of $\varepsilon_{\|}$ is higher than the value used in Fig. 8, whereas for both figures the same procedure for obtaining the remaining material parameters was followed. It turns out that a high value of $\varepsilon_{\|}$corresponds to high values of the anchoring constant. The effect of weak anchoring, i.e., of the splay-bend surface elasticity, is therefore less pronounced in the case of higher values of $\varepsilon_{\|}$.

Data points calculated from the measured capacitances and theoretical curves for the cell with $3.76 \mu \mathrm{m}$ cell gap are shown in Figs. 11 and 12. The attendant values of $\varepsilon_{\|}$ are $1 \%$ and $2 \%$ below $\varepsilon_{\|}^{\infty}=8.555$, respectively. The remaining material parameters are found in an analogous fashion as for the $2.82 \mu \mathrm{m}$ cell. The surface tilt angle below the Fréedericksz threshold agrees with the independently measured pretilt angle $\theta_{p}=1.18 \pm 0.10^{\circ}$.

The agreement between the theory and the capacitive measurements seems to be optimal with a splay-bend elastic constant $K_{13}=0 \mathrm{pN}$. This can be seen quite

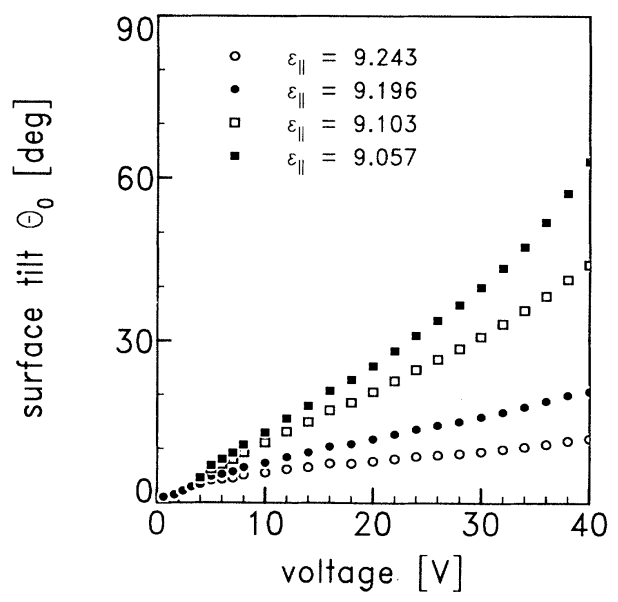

FIG. 9. Results for the tilt at the surface $\theta_{s}$ in the $2.82 \mu \mathrm{m}$ thick cell as calculated from the measured capacitance data for various values of $\varepsilon_{\|}$. For the sake of clarity some data points at low voltages have been omitted. 


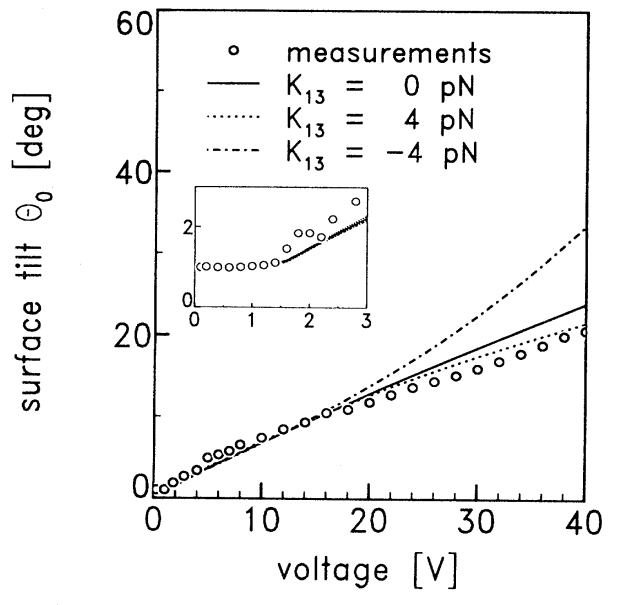

FIG. 10. Results for the surface tilt $\theta_{s}$ of the $2.82 \mu \mathrm{m}$ thick cell as calculated from the measured capacitance data for $\varepsilon_{\|}=9.196, \varepsilon_{\perp}=3.309, K_{11}=13.2 \mathrm{pN}$, and $K_{33}=18.3$ $\mathrm{pN}$. The lines give the results for the tilt at the surface as a function of the applied voltage for different values of $K_{13}$ and $C_{\theta}$, namely, for $K_{13}=4 \mathrm{pN}$ and $C_{\theta}=1390 \mu \mathrm{J} / \mathrm{m}^{2}$, for $K_{13}=0 \mathrm{pN}$ and $C_{\theta}=1110 \mu \mathrm{J} / \mathrm{m}^{2}$, and for $K_{13}=-4 \mathrm{pN}$ and $C_{\theta}=800 \mu \mathrm{J} / \mathrm{m}^{2}$.

clearly in Figs. 8 and 12, which depict the fits for both cells for the values of $\varepsilon_{\|} 2 \%$ below $\varepsilon_{\|}^{\infty}$. However, Figs. 10 and 11, which depict the fits for both cells for the values of $\varepsilon_{\|} 1 \%$ below $\varepsilon_{\|}^{\infty}$, do not allow for such a clear conclusion. Here, a small positive value of $K_{13}$ is possible as well. The reason for this indeterminacy is that the effects of weak anchoring are less pronounced in Figs. 10

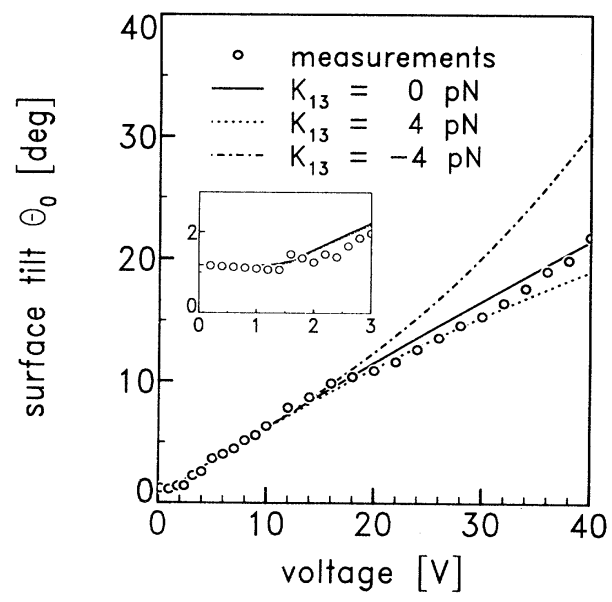

FIG. 11. Results for the tilt at the surface $\theta_{s}$ of the $3.76 \mu \mathrm{m}$ thick cell as calculated from the measured capacitance data for $\varepsilon_{\|}=8.469, \varepsilon_{\perp}=3.133, K_{11}=11.8 \mathrm{pN}$, and $K_{33}=16.4$ $\mathrm{pN}$. The curves give the results for the surface tilt as a function of the applied voltage as calculated for different values of $K_{13}$ and $C_{\theta}$, namely, for $K_{13}=4 \mathrm{pN}$ and $C_{\theta}=630 \mu \mathrm{J} / \mathrm{m}^{2}$, for $K_{13}=0 \mathrm{pN}$ and $C_{\theta}=470 \mu \mathrm{J} / \mathrm{m}^{2}$, and for $K_{13}=-4 \mathrm{pN}$ and $C_{\theta}=310 \mu \mathrm{J} / \mathrm{m}^{2}$.

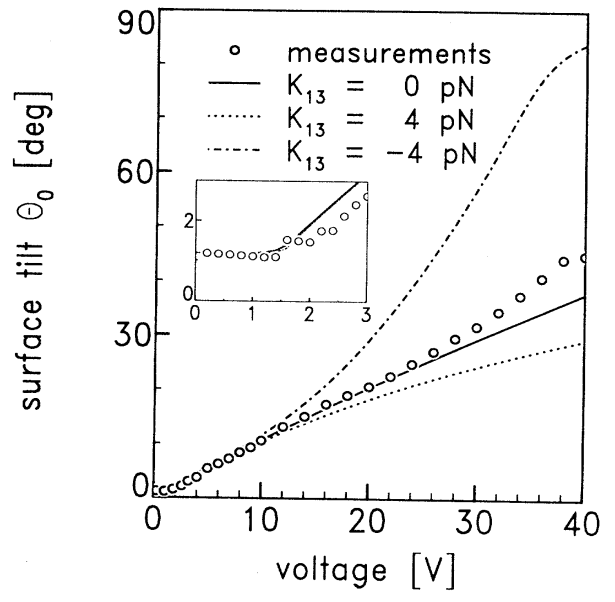

FIG. 12. Results for the surface tilt $\theta_{s}$ of the $3.76 \mu \mathrm{m}$ thick cell as calculated from the measured capacitance data for $\varepsilon_{\|}=8.384, \varepsilon_{\perp}=3.133, K_{11}=11.9 \mathrm{pN}$, and $K_{33}=16.5 \mathrm{pN}$. The lines give the results for the tilt at the surface as a function of the applied voltage as calculated for different values of $K_{13}$ and $C_{\theta}$, namely, for $K_{13}=4 \mathrm{pN}$ and $C_{\theta}=1220 \mu \mathrm{J} / \mathrm{m}^{2}$, for $K_{13}=0 \mathrm{pN}$ and $C_{\theta}=910 \mu \mathrm{J} / \mathrm{m}^{2}$, and for $K_{13}=-4 \mathrm{pN}$ and $C_{\theta}=620 \mu \mathrm{J} / \mathrm{m}^{2}$.

and 11 than they are in Figs. 8 and 12. Our method has two weaknesses. First, the anchoring strength appears to be quite high. As a consequence, relatively high voltages are needed to detect possible effects of $K_{13}$. Second, a different choice of $\varepsilon_{\|}$influences the quality of the fit, as is apparent from a comparison of, e.g., Figs. 8 and 10.

The measurements of the optical retardation were analyzed in a similar way. The surface tilt as derived from the optical data drops below $0^{\circ}$ at voltages above $6 \mathrm{~V}$. This is at variance with the $\theta_{s}$ values that were obtained from the capacitive data (Figs. 8-12) and it also contradicts the behavior described in Secs. II and IV. The retardation is observed to be a linear function of the inverse of the dielectric displacement $D$ at intermediate voltages as in [23]. However, extrapolation of this linear fit to infinite $D$ gives a finite, positive retardation at infinite voltage, whereas a finite, negative retardation is expected [23]. Figure 13 gives a comparison of the measured retardation and the calculated retardation on the assumption of strong anchoring. The material and cell parameters were determined from the measurements at low voltages. The curves calculated in the case of weak anchoring are below the curve for strong anchoring, as weak anchoring leads to a higher surface tilt. So, by varying the coefficients of the surface free energy $\left(C_{\theta}\right.$, $C_{2 \theta}$, and $K_{13}$ ) agreement with the measured retardation values cannot be achieved. A variation of the other material and cell parameters within reasonable limits does not give a satisfactory fit for both the low and the high voltages. Systematic deviations from the theoretical formula (12) due to multiple reflections [24] were estimated to be less than $1^{\circ}$, i.e., negligible. The difference might be due to flexoelectricity [25]. We incorporated this effect 


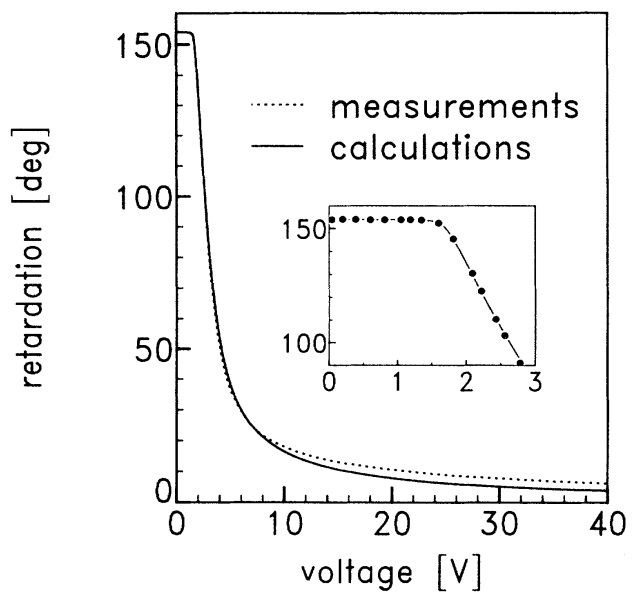

FIG. 13. Measured retardation data points for the cell with the $2.82 \mu \mathrm{m}$ cell gap. The curve gives the calculated retardation for an infinite anchoring constant. The other parameters are $K_{11}=13.0 \mathrm{pN}, K_{33}=18.0 \mathrm{pN}, \varepsilon_{\perp}=3.31$, and $\varepsilon_{\|}=9.10$.

into our model and assumed a value of the flexoelectric coefficient equal to $10 \mathrm{pC} / \mathrm{m}$, i.e., of the same order of magnitude as previously reported values for this coefficient $[26,27]$. It turned out that the effect is too small to account for the observed behavior. We conclude that the discrepancy with respect to the measured results cannot be explained by the present model. The measurement of the optical retardation for thin, nontwisted cells as a function of the applied voltage is a technique that is very sensitive to the director field near the substrate surfaces, as - at high voltages - the central, upright part of the director profile does not contribute to the optical retardation. In this respect, the optical measurements are more sensitive than the dielectric measurements and therefore the lack of agreement between theory and experiment is important.

Gleeson and Palffy-Muhoray $[20,21]$ performed the same type of experiments on low pretilt ECB cells filled with the liquid-crystal $5 \mathrm{CB}$ in order to determine the anchoring energy as a function of the surface tilt. There were two reasons for carrying out this type of measurement again in this study, instead of just using the data measured by Gleeson and Palffy-Muhoray. First, the experiments in [20] were performed on relatively thick liquid-crystal cells, in which the influence of surface elasticity is expected to be small. Secondly, very accurate values of the liquid-crystal and cell parameters were required for the calculation of the surface tilt from the measured data. We were able to measure these values in separate experiments for our own liquid-crystal cells.

The analysis of Gleeson and Palffy-Muhoray to find the surface tilt as a function of the applied voltage suffered the same problems [21] with the sensitivity to small variations in the liquid-crystal and cell parameters as the present analysis. Their results for the voltage dependence of the surface tilt [20] were fitted using our computer program. The results are shown in Fig. 14. Best agreement with the experimental results is obtained with $K_{13}=0$.

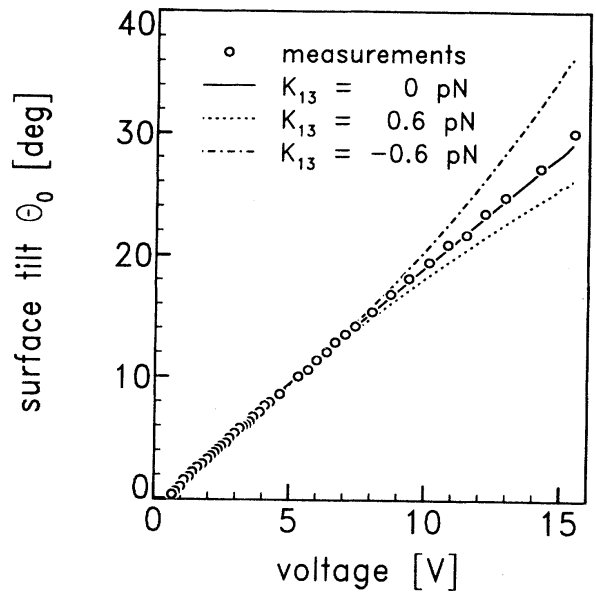

FIG. 14. Surface tilt as a function of the applied voltage of a $38 \mu \mathrm{m}$ thick cell filled with the liquid crystal 5CB as reported by J. T. Gleeson and P. Palffy-Muhoray, Liq. Cryst. 5, 663 (1989). The relevant parameters are $\varepsilon_{\perp}=9.93$, $\varepsilon_{\|}=18.62, K_{11}=3.0 \mathrm{pN}$, and $K_{33}=3.5 \mathrm{pN}$. The lines represent the calculated surface tilt as a function of the applied voltage for these parameters and for different values of $K_{13}$ and $C_{\theta}$, namely, for $K_{13}=0.6 \mathrm{pN}$ and $C_{\theta}=18.9 \mu \mathrm{J} / \mathrm{m}^{2}$, for $K_{13}=0 \mathrm{pN}$ and $C_{\theta}=16.0 \mu \mathrm{J} / \mathrm{m}^{2}$, and for $K_{13}=-0.6 \mathrm{pN}$ and $C_{\theta}=13.3 \mu \mathrm{J} / \mathrm{m}^{2}$. The pretilt was taken to be $\theta_{p}=0.1^{\circ}$.

The value $K_{13}=-0.2 K_{11}=-0.6 \mathrm{pN}$, which has been reported by Lavrentovich and Pergamenshchik [28], gave a less satisfactory fit. Lavrentovich and Pergamenshchik determined this value in experiments on hybridly aligned cells filled with the liquid-crystal material 5CB. Their analysis was made within the framework of the Pergamenshchik approach to surface elasticity. However, their analysis cannot be considered conclusive, as the difference between the splay and bend elastic constant is neglected.

Finally, we note that objections could be made from a fundamental point of view. The molecular ordering at the surface is different from that in the bulk, i.e., there is a spatial variation in the uniaxial order parameter [29], possibly biaxial order [30], and probably polar order $[29,31]$ in a thin layer near the surface. The consequences of this different ordering of the molecules for the validity of the continuum description of thin cells is, at present, not clear.

\section{CONCLUSION}

The elastic free energy of a nematic liquid crystal contains two surface elastic constants. According to the traditional analysis of nematic liquid crystals, these surface elastic constants may be neglected. In the case of weak anchoring, this is not correct. Currently there are two competing theories of surface elasticity in nematic liquid crystals, one put forward by Pergamenshchik [2], and one by Faetti [3]. As far as ECB cells are concerned, only the 
surface elastic constant $K_{13}$ is relevant. According to the Pergamenshchik theory this surface elastic constant has a profound influence on the voltage dependence of the surface tilt, the capacitance, and the optical retardation, provided that the anchoring constant is small. The magnitude of this influence is comparable to that of the influence of the bulk elastic constants. In general, the influence of variations of $K_{13}$ within Pergamenshchik's approach can be summarized as follows. At low voltages the surface tilt increases if $K_{13}$ is increased, whereas at high voltages the surface tilt decreases if $K_{13}$ is increased. According to the Faetti theory the surface elastic constant $K_{13}$ is irrelevant to ECB cells, i.e., the conventional theory is correct for ECB cells.

These different predictions make it possible to discriminate experimentally between both theories. The surface tilt as a function of the applied voltage for a thin cell filled with the liquid crystal ZLI 4792 can be obtained from the measured optical retardation and capacitance. The measured voltage dependence of the retardation cannot be fully described by the present model. This lack of agreement is an important problem. Best agreement between the results obtained from the measured capacitance and the theoretical curves is found with values of $K_{13}$ close to zero. A zero value for $K_{13}$ indicates that the Pergamenshchik theory is not valid. It turns out that the effect of $K_{13}$ on the theoretical curves is comparable in magnitude with the effect of variations in the remaining liquid-crystal and cell parameters within their experimental error.

\section{ACKNOWLEDGMENTS}

We are grateful to Peter Palffy-Muhoray (Kent State University) and Jim Gleeson (University of Calgary) for communication on surface tilt measurements. We acknowledge the stimulating support of Ger Vertogen (University of Nijmegen). Willem Potze (Philips Research) is thanked for suggesting the mathematical treatment described in Appendix A. We obtained helpful support in our computational work from Ronald van Rijn, Ad Leeuwestein, and Stephan Demmers (Philips Research). We are indebted to Gerda van de Spijker (Philips Research) for supplying us with liquid-crystal cells.

\section{APPENDIX A}

The spatial derivative of the tilt angle at $z=0$ follows from the Euler-Lagrange equation as

$$
\theta^{\prime}(0)=h\left(\theta_{s}, \theta_{m}, D\right)
$$

with

$$
\begin{aligned}
h\left(\theta_{s}, \theta_{m}, D\right) & =\left[\frac{\left(\cos ^{2} \theta_{s}-\cos ^{2} \theta_{m}\right) g\left(\theta_{s}, \theta_{m}\right)}{1-\gamma \cos ^{2} \theta_{s}}\right]^{1 / 2} \\
& =D \sqrt{\frac{\alpha\left(\cos ^{2} \theta_{s}-\cos ^{2} \theta_{m}\right)}{\varepsilon_{0} \varepsilon_{\|} K_{33}\left(1-\gamma \cos ^{2} \theta_{s}\right)\left(1-\alpha \cos ^{2} \theta_{s}\right)\left(1-\alpha \cos ^{2} \theta_{m}\right)}}
\end{aligned}
$$

The maximum tilt angle $\theta_{m}$ and the $z$ component of the displacement field $D$ are implicitly defined as a function of the surface tilt $\theta_{s}$ by means of Eqs. (7) and (9), which can be written as

$$
\begin{aligned}
& \frac{d}{2}=f_{1}\left(\theta_{s}, \theta_{m}, D\right), \\
& \frac{V}{2}=f_{2}\left(\theta_{s}, \theta_{m}, D\right),
\end{aligned}
$$

with the functions

$$
\begin{aligned}
f_{1}\left(\theta_{s}, \theta_{m}, D\right)= & \sqrt{\frac{\varepsilon_{0} \varepsilon_{\|} K_{33}\left(1-\alpha \cos ^{2} \theta_{m}\right)}{\alpha D}} \\
& \times \int_{\theta_{s}}^{\theta_{m}} \frac{d \theta \sqrt{1-\gamma \cos ^{2} \theta}}{\sqrt{\cos ^{2} \theta-\cos ^{2} \theta_{m}}} \sqrt{1-\alpha \cos ^{2} \theta}
\end{aligned}
$$

$$
\begin{aligned}
f_{2}\left(\theta_{s}, \theta_{m}, D\right)= & \sqrt{\frac{K_{33}\left(1-\alpha \cos ^{2} \theta_{m}\right)}{\varepsilon_{0} \varepsilon_{\|} \alpha}} \\
& \times \int_{\theta_{s}}^{\theta_{m}} \frac{d \theta \sqrt{1-\gamma \cos ^{2} \theta}}{\sqrt{\cos ^{2} \theta-\cos ^{2} \theta_{m}}} \frac{1}{\sqrt{1-\alpha \cos ^{2} \theta}}
\end{aligned}
$$

The derivative of $\theta^{\prime}(0)$ with respect to $\theta_{s}$ can thus be expressed as

$$
\frac{d \theta^{\prime}(0)}{d \theta_{s}}=\frac{\partial h}{\partial \theta_{s}}+\frac{\partial h}{\partial \theta_{m}} \frac{d \theta_{m}}{d \theta_{s}}+\frac{\partial h}{\partial D} \frac{d D}{d \theta_{s}},
$$

with the partial derivatives: 


$$
\begin{aligned}
\frac{\partial h}{\partial \theta_{s}}= & -\left(\frac{1}{\cos ^{2} \theta_{s}-\cos ^{2} \theta_{m}}+\frac{\gamma}{1-\gamma \cos ^{2} \theta_{s}}\right. \\
& \left.+\frac{\alpha}{1-\alpha \cos ^{2} \theta_{s}}\right) \sin \theta_{s} \cos \theta_{s} \theta^{\prime}(0) \\
\frac{\partial h}{\partial \theta_{m}}= & \left(\frac{1}{\cos ^{2} \theta_{s}-\cos ^{2} \theta_{m}}-\frac{\alpha}{1-\alpha \cos ^{2} \theta_{m}}\right) \\
& \times \sin \theta_{m} \cos \theta_{m} \theta^{\prime}(0) \\
\frac{\partial h}{\partial D}= & \frac{\theta^{\prime}(0)}{D}
\end{aligned}
$$

Expressions for the derivatives of $\theta_{m}$ and $D$ with respect to $\theta_{s}$ can be derived in the following way. Equations (A4) have a solution for all $\theta_{s}$. Clearly

$$
\begin{aligned}
\frac{d f_{i}}{d \theta_{s}} & =\frac{\partial f_{i}}{\partial \theta_{s}}+\frac{\partial f_{i}}{\partial \theta_{m}} \frac{d \theta_{m}}{d \theta_{s}}+\frac{\partial f_{i}}{\partial D} \frac{d D}{d \theta_{s}} \quad i=1,2 . \\
& =0,
\end{aligned}
$$

The derivatives of the functions $f_{i}$ with respect to $D$ can be written as

$$
\begin{aligned}
& \frac{\partial f_{1}}{\partial D}=-\frac{d}{2 D} \\
& \frac{\partial f_{2}}{\partial D}=0
\end{aligned}
$$

The derivatives of the functions $f_{i}$ with respect to $\theta_{s}$ are

$$
\begin{aligned}
& \frac{\partial f_{1}}{\partial \theta_{s}}=-\frac{1}{\theta^{\prime}(0)}, \\
& \frac{\partial f_{2}}{\partial \theta_{s}}=-\frac{D}{\varepsilon_{0} \varepsilon_{\|}\left(1-\alpha \cos ^{2} \theta_{s}\right) \theta^{\prime}(0)} .
\end{aligned}
$$

The expressions for the derivatives of the functions $f_{i}$ with respect to $\theta_{m}$ are more complicated, as the integrand in the expressions for $f_{i}$ is singular in $\theta=\theta_{m}$. This singularity can be dealt with by a suitable substitution of variables, such as

$$
\sin \theta=\sin \theta_{m} \sin \psi
$$

The functions $f_{i}$ can now be expressed in a nonsingular manner:

$$
\begin{aligned}
f_{1}= & \sqrt{\frac{\varepsilon_{0} \varepsilon_{\|} K_{33}\left(1-\alpha \cos ^{2} \theta_{m}\right)}{\alpha D}} \\
& \times \int_{\psi_{0}}^{\pi / 2} d \psi \sqrt{\frac{1-\gamma\left(1-\sin ^{2} \theta_{m} \sin ^{2} \psi\right)}{1-\sin ^{2} \theta_{m} \sin ^{2} \psi}} \\
& \times \sqrt{1-\alpha\left(1-\sin ^{2} \theta_{m} \sin ^{2} \psi\right)}, \\
f_{2}= & \sqrt{\frac{K_{33}\left(1-\alpha \cos ^{2} \theta_{m}\right)}{\varepsilon_{0} \varepsilon_{\|} \alpha}} \\
& \times \int_{\psi_{0}}^{\pi / 2} d \psi \sqrt{\frac{1-\gamma\left(1-\sin ^{2} \theta_{m} \sin ^{2} \psi\right)}{1-\sin ^{2} \theta_{m} \sin ^{2} \psi}} \\
& \times \frac{1}{\sqrt{1-\alpha\left(1-\sin ^{2} \theta_{m} \sin ^{2} \psi\right)}} .
\end{aligned}
$$

After a tedious exercise in differentiating we have

$$
\begin{aligned}
\frac{\partial f_{1}}{\partial \theta_{m}}= & \frac{\tan \theta_{s}}{\tan \theta_{m} \theta^{\prime}(0)}+\frac{d \alpha \sin \theta_{m} \cos \theta_{m}}{2\left(1-\alpha \cos ^{2} \theta_{m}\right)} \\
& +\sqrt{\frac{\varepsilon_{0} \varepsilon_{\|} K_{33}\left(1-\alpha \cos ^{2} \theta_{m}\right)}{\alpha D}} I_{1} \\
\frac{\partial f_{2}}{\partial \theta_{m}}= & \frac{D \tan \theta_{s}}{\varepsilon_{0} \varepsilon_{\|}\left(1-\alpha \cos ^{2} \theta_{s}\right) \tan \theta_{m} \theta^{\prime}(0)} \\
& +\frac{V \alpha \sin \theta_{m} \cos \theta_{m}}{2\left(1-\alpha \cos ^{2} \theta_{m}\right)} \\
& +\sqrt{\frac{K_{33}\left(1-\alpha \cos ^{2} \theta_{m}\right)}{\varepsilon_{0} \varepsilon_{\|} \alpha}} I_{2},
\end{aligned}
$$

with

$$
\begin{aligned}
I_{1}= & \int_{\theta_{s}}^{\theta_{m}} \frac{d \theta \sqrt{1-\gamma \cos ^{2} \theta}}{\sqrt{\cos ^{2} \theta_{s}-\cos ^{2} \theta_{m}}} \sqrt{1-\alpha \cos ^{2} \theta} \\
& \times\left(\frac{\gamma}{1-\gamma \cos ^{2} \theta}+\frac{\alpha}{1-\alpha \cos ^{2} \theta}+\frac{1}{\cos ^{2} \theta}\right) \\
& \times \frac{\sin ^{2} \theta}{\tan \theta_{m}}, \\
I_{2}= & \int_{\theta_{s}}^{\theta_{m}} \frac{d \theta \sqrt{1-\gamma \cos ^{2} \theta}}{\sqrt{\cos ^{2} \theta_{s}-\cos ^{2} \theta_{m}}} \frac{1}{\sqrt{1-\alpha \cos ^{2} \theta}} \\
& \times\left(\frac{\gamma}{1-\gamma \cos ^{2} \theta}-\frac{\alpha}{1-\alpha \cos ^{2} \theta}+\frac{1}{\cos ^{2} \theta}\right) \\
& \times \frac{\sin { }^{2} \theta}{\tan \theta_{m}} .
\end{aligned}
$$

Now it follows that

$$
\begin{aligned}
\frac{d \theta_{m}}{d \theta_{s}}= & -\frac{\partial f_{2}}{\partial \theta_{s}} / \frac{\partial f_{2}}{\partial \theta_{m}} \\
= & {\left[\frac{\tan \theta_{s}}{\tan \theta_{m}}+\frac{\varepsilon_{0} \varepsilon_{\|} V \alpha \sin \theta_{m} \cos \theta_{m}\left(1-\alpha \cos ^{2} \theta_{s}\right) \theta^{\prime}(0)}{2 D\left(1-\alpha \cos ^{2} \theta_{m}\right)}\right.} \\
& \left.+\sqrt{\frac{\varepsilon_{0} \varepsilon_{\|} K_{33}\left(1-\alpha \cos ^{2} \theta_{m}\right)}{\alpha D}}\left(1-\alpha \cos ^{2} \theta_{s}\right) \theta^{\prime}(0) I_{2}\right]^{-1}
\end{aligned}
$$




$$
\begin{aligned}
& \frac{d D}{d \theta_{s}}=\left(-\frac{\partial f_{1}}{\partial \theta_{s}}-\frac{\partial f_{1}}{\partial \theta_{m}} \frac{d \theta_{m}}{d \theta_{s}}\right) / \frac{\partial f_{1}}{\partial D} \\
&=\frac{2 D}{\theta^{\prime}(0) d}\left\{\left[\frac{\tan \theta_{s}}{\tan \theta_{m}}+\frac{d \alpha \sin \theta_{m} \cos \theta_{m} \theta^{\prime}(0)}{2\left(1-\alpha \cos ^{2} \theta_{m}\right)}+\sqrt{\frac{\varepsilon_{0} \varepsilon_{\|} K_{33}\left(1-\alpha \cos ^{2} \theta_{m}\right)}{\alpha D}} I_{1}\right]\right. \\
& \times\left[\frac{\tan \theta_{s}}{\tan \theta_{m}}+\frac{\varepsilon_{0} \varepsilon_{\|} V \alpha \sin \theta_{m} \cos \theta_{m}\left(1-\alpha \cos ^{2} \theta_{s}\right) \theta^{\prime}(0)}{2 D\left(1-\alpha \cos ^{2} \theta_{m}\right)}\right. \\
&\left.\left.+\sqrt{\frac{\varepsilon_{0} \varepsilon_{\|} K_{33}\left(1-\alpha \cos ^{2} \theta_{m}\right)}{\alpha D}}\left(1-\alpha \cos ^{2} \theta_{s}\right) \theta^{\prime}(0) I_{2}\right]^{-1}-1\right\} .
\end{aligned}
$$

Finally, the expression for the derivative of $\theta^{\prime}(0)$ with respect to $\theta_{s}$ can be written as

$$
\begin{aligned}
\frac{d \theta^{\prime}(0)}{d \theta_{s}}= & -\left(\frac{1}{\cos ^{2} \theta_{s}-\cos ^{2} \theta_{m}}+\frac{\gamma}{1-\gamma \cos ^{2} \theta_{s}}+\frac{\alpha}{1-\alpha \cos ^{2} \theta_{s}}\right) \sin \theta_{s} \cos \theta_{s} \theta^{\prime}(0) \\
& +\left\{\frac{\sin \theta_{m} \cos \theta_{m}}{\cos ^{2} \theta_{s}-\cos ^{2} \theta_{m}}-\frac{\varepsilon_{0} \varepsilon_{\|} V \alpha \sin \theta_{m} \cos \theta_{m}\left(1-\alpha \cos ^{2} \theta_{s}\right)}{2 D\left(1-\alpha \cos ^{2} \theta_{m}\right)}\right. \\
& \left.+\frac{2}{d} \sqrt{\frac{\varepsilon_{0} \varepsilon_{\|} K_{33}\left(1-\alpha \cos ^{2} \theta_{m}\right)}{\alpha D}}\left[I_{1}-\left(1-\alpha \cos ^{2} \theta_{s}\right) I_{2}\right]\right\} \theta^{\prime}(0) \\
& \times\left\{\frac{\tan \theta_{s}}{\tan \theta_{m}}+\frac{\varepsilon_{0} \varepsilon_{\|} V \alpha \sin \theta_{m} \cos \theta_{m}\left(1-\alpha \cos ^{2} \theta_{s}\right) \theta^{\prime}(0)}{2 D\left(1-\alpha \cos ^{2} \theta_{m}\right)}\right. \\
& \left.+\sqrt{\frac{\varepsilon_{0} \varepsilon_{\|} K_{33}\left(1-\alpha \cos ^{2} \theta_{m}\right)}{\alpha D}}\left(1-\alpha \cos ^{2} \theta_{s}\right) \theta^{\prime}(0) I_{2}\right\}^{-1} .
\end{aligned}
$$

\section{APPENDIX B}

In order to gain a qualitative understanding of the results of Sec. III we analyzed the behavior of low pretilt ECB cells at low voltages. Then the surface tilt $\theta_{s}$ is small. It follows that the total free energy up to second order in $\theta_{s}$ is given by

$$
\begin{aligned}
F= & \frac{1-\alpha}{2 \alpha} K_{11} q^{2} d+C_{\theta} \theta_{p}^{2}-2 C_{\theta} \theta_{p} \theta_{s} \\
& +\left[C_{\theta}-\left(K_{11}+2 K_{13}\right) q \tan (q d / 2)\right] \theta_{s}^{2},
\end{aligned}
$$

where $q$ is related to the applied voltage by

$$
V=\sqrt{\frac{K_{11}}{\varepsilon_{0}\left(\varepsilon_{\|}-\varepsilon_{\perp}\right)}} q d .
$$

Minimizing $F$ with respect to $\theta_{s}$ gives the following expression for $\theta_{s}$ :

$$
\theta_{s}=\frac{C_{\theta} \theta_{p}}{C_{\theta}-\left(K_{11}+2 K_{13}\right) q \tan (q d / 2)}
$$

Clearly $\theta_{s}$ increases with $K_{13}$, in accordance with the results of Sec. III. The term linear in $\theta_{s}$ in (B1) does not appear when $\theta_{p}=0$. Then the surface tilt $\theta_{s}$ will become nonzero when the coefficient of the quadratic term in (B1) becomes negative. Consequently the Fréedericksz transition is only sharp when $\theta_{p}=0$. The threshold for the Fréedericksz transition follows from

$$
\cot (q d / 2)=\frac{K_{11}+2 K_{13}}{C_{\theta}} q .
$$

The strong anchoring value threshold voltage corresponds to $q d=\pi$, which is found in the limit $C_{\theta} d /\left(K_{11}+\right.$ $\left.2 K_{13}\right) \rightarrow \infty$. We infer that the parameter $C_{\theta} d / K$, where $K$ is a typical elastic constant, determines the strength of the anchoring. This can also be understood from a purely dimensional argument, as the ratio between the anchoring term and the elastic terms is equal to $C_{\theta} d / K$ within a factor of the order of unity.

A similar analysis can be made of the high-voltage behavior. If $\theta_{s}$ deviates slightly from $\pi / 2$ then the total free energy can be expanded in the difference $\pi / 2-\theta_{s}$ :

$$
\begin{aligned}
F= & \frac{K_{33} q^{2} d}{2 \alpha}+C_{\theta} \cos ^{2} \theta_{p}-C_{\theta} \sin \left(2 \theta_{p}\right)\left(\frac{\pi}{2}-\theta_{s}\right) \\
& +\left[\left(K_{33}-2 K_{13}\right) q \tanh (q d / 2)-C_{\theta} \cos \left(2 \theta_{p}\right)\right] \\
& \times\left(\frac{\pi}{2}-\theta_{s}\right)^{2},
\end{aligned}
$$

where $q$ is related to the applied voltage by

$$
V=\sqrt{\frac{K_{33}}{\varepsilon_{0}\left(\varepsilon_{\|}-\varepsilon_{\perp}\right)}} q d .
$$


Minimizing $F$ with respect to $\theta_{s}$ gives the following expression for $\theta_{s}$ :

$$
\theta_{s}=\frac{\pi}{2}-\frac{C_{\theta} \sin \left(2 \theta_{p}\right) / 2}{\left(K_{33}-2 K_{13}\right) q \tanh (q d / 2)-C_{\theta} \cos \left(2 \theta_{p}\right)} .
$$

Clearly $\theta_{s}$ decreases with $K_{13}$, in accordance with the results of Sec. III. The term linear in $\theta_{s}$ in (B5) does not appear when $\theta_{p}=0$, i.e., when the anchoring energy has a maximum at $\theta_{s}=\pi / 2$. Then the surface tilt $\theta_{s}$ will become equal to $\pi / 2$ when the coefficient of the quadratic term in (B5) becomes positive. Consequently there is a saturation transition only when $\theta_{p}=0$. The threshold for this saturation transition follows from

$$
\operatorname{coth}(q d / 2)=\frac{K_{33}-2 K_{13}}{C_{\theta}} q
$$

In case of strong anchoring, i.e., in the limit $C_{\theta} d /\left(K_{33}-\right.$ $\left.2 K_{13}\right) \rightarrow \infty$, this equation has no solution. This means that in the limit of strong anchoring there is no saturation transition at a finite voltage. We want to emphasize that the Rapini-Papoular approximation of the anchoring energy is only correct when the surface tilt angle deviates slightly from the pretilt angle. The results for the saturation regime can therefore only be qualitatively correct.
[1] C. Oldano and G. Barbero, J. Phys. (Paris) Lett. 46, 451 (1985); Phys. Lett. 110A, 213 (1985); G. Barbero and C. Oldano, Nuovo Cimento D 6, 479 (1985).

[2] V. M. Pergamenshchik, Phys. Rev. E 48, 1254 (1993).

[3] S. Faetti, Phys Rev. E 49, 5332 (1994); 49, 4192 (1994).

[4] E. Kaneko, Liquid Crystal TV Displays: Principles and Applications of Liquid Crystal Displays (KTK Scientific Publishers, Tokyo, 1987).

[5] P. I. C. Teixeira, V. M. Pergamenshchik, and T. J. Sluckin, Mol. Phys. 80, 1339 (1993).

[6] H. A. van Sprang, J. Appl. Phys. 71, 4826 (1992).

[7] C. W. Oseen, Trans. Faraday Soc. 29, 883 (1933).

[8] F. C. Frank, Discuss. Faraday Soc. 25, 19 (1958).

[9] J. Nehring and A. Saupe, J. Chem. Phys. 54, 337 (1971).

[10] A. Rapini and M. Papoular, J. Phys. (Paris) Colloq. 30, C4-54 (1969).

[11] S. Stallinga and G. Vertogen, Phys. Rev. E 53, 1692 (1996).

[12] V. M. Pergamenshchik, P. I. C. Teixeira, and T. J. Sluckin, Phys Rev. E 48, 1265 (1993).

[13] J. Nehring, A. R. Kmetz, and T. J. Scheffer, J. Appl. Phys. 47, 850 (1976).

[14] K. H. Yang, Jpn. J. Appl. Phys. 22, 389 (1983); K. H. Yang and C. Rosenblatt, Appl. Phys. Lett. 43, 62 (1983).

[15] S. Shimoda, IH. Mada, and S. Kobayashi, Jpn. J. Appl. Phys. 17, 1359 (1978).

[16] T. Motooka and A. Fukuhara, J. Appl. Phys. 50, 6907 (1979).

[17] M. Nishikawa et al., in Proceedings of the International
Display Research Conference, Hiroshima, Japan, 1992, edited by S. Kobayashi, M. Fukushima, and T. Uchida (Society for Information Display, Playa del Rey, 1992), p. 819.

[18] N. A. J. M. van Aerle and A. J. W. Tol, Macromolecules 27, 6520 (1994).

[19] H. A. van Sprang, Mol. Cryst. Liq. Cryst. 199, 19 (1991).

[20] J. T. Gleeson and P. Palffy-Muhoray, Liq. Cryst. 5, 663 (1989).

[21] J. T. Gleeson, Master's thesis, University of British Columbia, 1988.

[22] K. Tarumi (E. Merck, Darmstadt, Germany) (private communication).

[23] H. Yokoyama and H. A. van Sprang, J. Appl. Phys. 57, 4520 (1985).

[24] P. Palffy-Muhoray (private communication).

[25] G. Barbero (private communication).

[26] G. Barbero and G. Durand, J. Phys. (Paris) 47, 2129 (1986).

[27] P. R. Maheswara Murthy, V. A. Raghunathan, and N. V. Madhusudana, Liq. Cryst. 14, 483 (1993).

[28] O. D. Lavrentovich and V. M. Pergamenshchik, Phys. Rev. Lett. 73, 979 (1994).

[29] B. Jérôme, Rep. Prog. Phys. 54, 391 (1991).

[30] N. Kothekar, D. W. Allender, and R. M. Hornreich, Phys. Rev. E 49, 2150 (1994).

[31] P. Guyot-Sionnest, H. Hsiung, and Y. R. Shen, Phys. Rev. Lett. 57, 2963 (1986). 